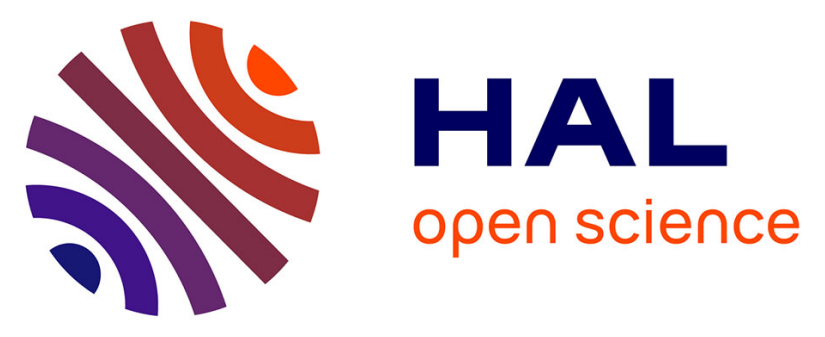

\title{
Group RNTI for multi-users multiplexing radio voice transmission for enhancing voice capacity over LTE in PMR context
}

Hang Nguyen, Manh Cuong Nguyen, Eric Georgeaux, Philippe Mège, Laurent Martinod

\section{To cite this version:}

Hang Nguyen, Manh Cuong Nguyen, Eric Georgeaux, Philippe Mège, Laurent Martinod. Group RNTI for multi-users multiplexing radio voice transmission for enhancing voice capacity over LTE in PMR context. VTC Spring 2015 : 81st Vehicular Technology Conference, May 2015, Glasgow, United Kingdom. pp.1 - 5, 10.1109/VTCSpring.2015.7145854 . hal-01262418

\section{HAL Id: hal-01262418 \\ https://hal.science/hal-01262418}

Submitted on 26 Jan 2016

HAL is a multi-disciplinary open access archive for the deposit and dissemination of scientific research documents, whether they are published or not. The documents may come from teaching and research institutions in France or abroad, or from public or private research centers.
L'archive ouverte pluridisciplinaire HAL, est destinée au dépôt et à la diffusion de documents scientifiques de niveau recherche, publiés ou non, émanant des établissements d'enseignement et de recherche français ou étrangers, des laboratoires publics ou privés. 


\title{
Group_RNTI for Multi-users Multiplexing Radio Voice Transmission for Enhancing Voice Capacity over LTE in PMR Context
}

\author{
Manh-Cuong Nguyen and Hang Nguyen ${ }^{1} \quad$ Eric Georgeaux, Philippe Mege and Laurent Martinod ${ }^{2}$
}

\begin{abstract}
The Multi-users Multiplexing Radio Voice Transmission method [1] is used for reducing the data overhead of Voice over Long Term Evolution (VoLTE) in the Professional Mobile Radio (PMR) context. In the Multi-users Multiplexing Radio Voice Transmission method, voice packets from different users having the same Modulation and Coding Scheme (MCS) can be clustered into one same LTE packet in the downlink transmission for reducing the data overhead caused by the difference between the LTE packet size and the PMR voice payload. However, in order to increase the voice capacity of VoLTE in PMR context both data overhead and control overhead have to be reduced. Therefore, in this paper, we will present a new Group Radio Network Identifier (Group_RNTI) method for reducing the control overhead in the multiplexing scheme. In the Group_RNTI method, UEs having the same Modulation and Coding Scheme (MCS) will be clustered in one or more groups. Each group is assigned a common Group_RNTI. Control information is sent for groups rather than for each UE. The results show that the Group_RNTI can reduce the control signaling up to $85 \%$ compared to the fully dynamic scheduling (FD) in the standard LTE in case of using Multi-users Multiplexing Radio Voice Transmission method. The use of Group_RNTI for the Multi-users Multiplexing Radio Voice Transmission method allows reducing both data overhead and control overhead issues for VoLTE in PMR context. The proposed method ensures an augmentation of voice capacity and a reduction of blocking rate for VoLTE in PMR context.
\end{abstract}

Index Terms-Group_RNTI, LTE, multiplexing, PMR, MCS

\section{INTRODUCTION}

Professional or Private Mobile Radio (PMR) networks provide compromising services for critical voice and data application [2]. Current PMR networks, such as Terrestrial Trunked Radio (TETRA), Association of Public Safety Communications Officials Project 25 (APCO25) still use second generation $(2 \mathrm{G})$ technologies, have to be replaced by new technologies to support new user demands [3]. Long Term Evolution (LTE) technology is chosen as one of the candidates for the next generation broadband PMR networks. However, LTE technology has to be adapted to fit the main criteria of the PMR networks. One of the most important criteria of PMR

\footnotetext{
${ }^{1}$ Institut Mines-Telecom, Telecom SudParis

Samovar Laboratory, UMR 5157, CNRS, Evry, France

\{manh_cuong.nguyen, hang.nguyen\}@it-sudparis.eu

${ }^{2}$ Department of Security and Communication Solutions CASSIDIAN (an EADS Company), Elancourt, France

\{eric.georgeaux, philippe.mege, laurent.martinod \}@ cassidian.com
}

This work was accomplished in the framework of the collaborative project SOAPS.2, labeled by the Systematic PARIS-REGION Cluster networks is the voice capacity in term number of users. But the LTE physical layer is not optimal for low bit rate voice communication in PMR context so that the voice capacity of LTE is not increased as expected of PMR providers. In the LTE network one pair of Physical Resource Blocks (PRBs) is the smallest User Assignment Unit. However, the smallest LTE packet size is still too large in case of low bit rate voice communication in the PMR context, especially when small voice payload is transmitted in the high quality LTE channel. This reduces the maximum number of packets that can be transmitted in a unit time. In addition, the limitation of the control plane caused by the shortage of L1 control channels that are used for the resource allocation in LTE is another factor that affects the voice capacity of the LTE. Several attempts have been made to increase the voice capacity over LTE. Some studies try to reduce the data overhead such as ROHC [4], CRC and error reduction [5], [6]. Some other studies try to reduce the control overhead of the Fully Dynamic (FD) scheduling such as Semi-persistent scheduling [7], [8] or group scheduling [9], [10]. Nevertheless, the existent methods focus mainly for high and medium voice payload over LTE (the code rate of voice coder is about $12.2 \mathrm{kbps}$ ). Few researches pay attention in the gap between the LTE packet size and the small voice payload in case of very small voice payload (Advanced MultiBand Excitation (AMBE) 2450 bps in case of PMR networks). Moreover, there are few papers that can solve both major issues of VoIP over LTE in PMR context: data overhead and control overhead.

Therefore, we have proposed a new Multi-users Multiplexing Radio Voice Transmission for Enhancing Voice Capacity over LTE in PMR context. This method can solve both overheads of voice over LTE downlink in PMR context. Our method clusters voice packets from different users into one same LTE packet in the downlink transmission for reducing the data overhead caused by the difference between the LTE packet size and the voice payload and applies a Group_RNTI method for reducing the control overhead. The architecture for our Multi-users Multiplexing Radio Voice Transmission method have been presented in [1]. In this paper, we will present the Group_RNTI method to solve the control overhead issue for the multiplexing scheme of VoLTE in PMR context. The Group_RNTI method clusters UEs having the same Modulation and Coding Scheme (MCS) into one or more groups. Each group is assigned a common Group_RNTI. Control information is sent for groups rather than for each 
Normal scenario

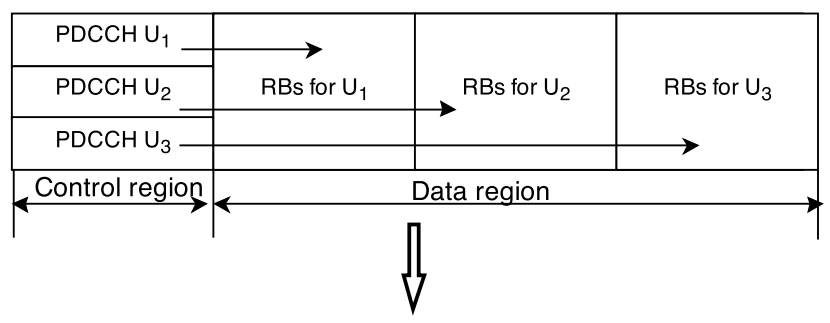

Control overhead scenario in the standard LTE

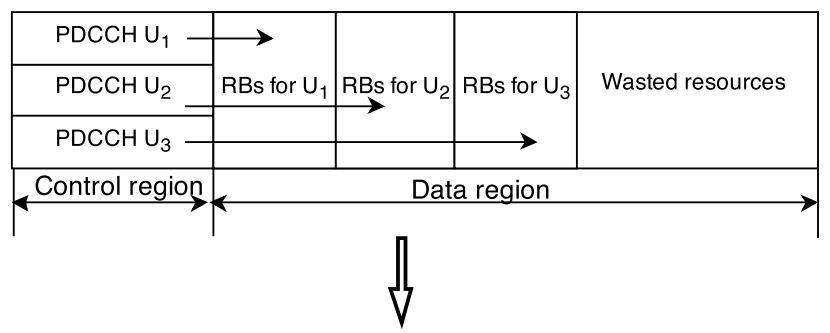

Control overhead in the Multiplexing scheme

\begin{tabular}{|c|c|c|}
\hline $\mathrm{PDCCH} \mathrm{U}_{1}$ & $\longrightarrow$ & \\
\hline $\mathrm{PDCCH} \mathrm{U}_{2}$ & RBs for $U_{1} \& U_{2} \& U_{3}$ & Wasted resources \\
\hline $\mathrm{PDCCH} \mathrm{U}_{3}$ & & \\
\hline Control region & \multicolumn{2}{|c|}{ Data region } \\
\hline
\end{tabular}

Fig. 1. Control overhead issue

UE. The details of this method is presented in section II. The performance of our method is evaluated in section III. Section IV gives the conclusion and some perspectives.

\section{GRoup RNTI FOR THE MULTI-USER MULTIPLEXING VOICE RADIO TRANSMISSION METHOD}

A. Control overhead in the Multi-users Multiplexing Radio Voice Transmission for Enhancing Voice Capacity

LTE uses Fully Dynamic (FD) as the base scheduler for resource allocation. In the FD scheduler, each data packet needs to associate with a $\mathrm{L} 1$ control signaling. When a large number of voice packets are served at a time, the number of required control channels is increased. However, the number of Physical Downlink Control Channel (PDCCH) is limited because the PDCCH channels can only use one to three OFDM symbols in each subframe to create the L1 signaling control channels. This can in-turn limit the number of simultaneous voice calls.

Figure 1 shows the control overhead issue in the Multi-users Multiplexing Radio Voice Transmission method. The control overhead issue in the Multi-user Multiplexing Voice Radio Transmission method is similar to that in the standard LTE. The control overhead occurs when there is not enough PDCCH channels for the scheduling. However, in the multiplexing scheme, UEs in a same group have the same allocation information (same DCI value) so that an adequate method for this situation needs to be considered.

\section{B. Principle of group_RNTI}

The principle of group_RNTI includes two steps: initialization of Group_RNTI and using of Group_RNTI for resource allocation.

In the first step, UEs having the same MCS values can be classified in one or more groups. Each group is assigned a common Group_RNTI. We assume that in the PMR context, the number of UEs in a cell is high so that we can cluster only UEs having the same MCS value. The initialization of group is done by a new Setup_Group_RNTI PDCCH. Setup_group_DCI combines with its CRC bits scrambled by the C_RNTI value of UE to create Setup_Group_RNTI PD$\mathrm{CCH}$. The structure of Setup_group_DCI is presented in table I. The Setup_Group_RNTI PDCCH is used for the first time resource allocation or when UE changes the group (changing of MCS value of UE).

TABLE I

SETUP_GROUP_DCI STRUCTURE

\begin{tabular}{|l|l|l|}
\hline Field Name & Length (bits) & Comment \\
\hline \multirow{2}{*}{ RBA } & $\begin{array}{l}5(1.4 \mathrm{MHz}) \\
7(3 \mathrm{MHz}) \\
9(5 \mathrm{MHz})\end{array}$ & Resource block assignment \\
& 5 & Modulation and Coding Scheme \\
\hline MCS & 5 & Identification of group \\
\hline Group_RNTI & 16 & Position of UE in the group \\
\hline Group_Position & 4 & \multicolumn{2}{|l}{} \\
\hline
\end{tabular}

TABLE II

GROUPMULTIPLEXING_DCI STRUCTURE

\begin{tabular}{|l|l|l|}
\hline Field Name & Length (bits) & Comment \\
\hline & $5(1.4 \mathrm{MHz})$ & \\
RBA & $7(3 \mathrm{MHz})$ & Resource block assignment \\
& $9(5 \mathrm{MHz})$ & \\
\hline MCS & 5 & Modulation and Coding Scheme \\
\hline Group Bitmap & 16 & Group information for UEs \\
\hline
\end{tabular}

In the second step, assume that at the $i^{\text {th }}$ TTI, there are $n$ voice payload of $n$ UEs that can be multiplexed into one LTE subframe. In this case, instead of sending $n$ different PDCCH channels for $n$ UEs having the same DCI value, eNodeB will send $k$ different Group_RNTI PDCCH channels for $k$ groups. The scheduler will try to cluster maximum possible the UEs in the same Group_RNTI. GroupMultiplexing_DCI combines with its CRC bits scrambled by the Group_RNTI value to create Group_RNTI PDCCH. The structure of GroupMultiplexing_DCI is presented in table II.

After having received a setup_group_DCI message, the UE starts monitoring DCI messages sent over the PDCCH scrambled by the Group_RNTI value. When a valid message is found, the bit at position Group_Position in the bitmap is checked: a ' 1 ' indicates that the $\mathrm{RB}(\mathrm{s})$ contain some data for that UE (see Figure 2). In our case, UE keeps both C_RNTI and Group_RNTI values. Group_RNTI is a temporary value. Group_RNTI can be changed if UE changes the group (changing of MCS). The Group_RNTI value in this case is assigned by eNodeB. 


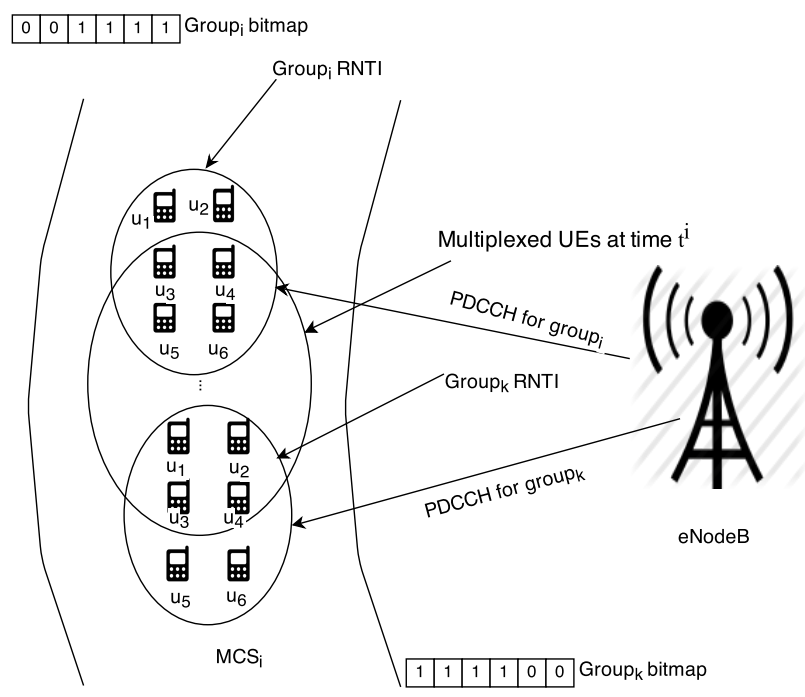

Fig. 2. Group_RNTI scheduling

\section{Group - Change management}

Assume that at $i^{t h}$ TTI, the quality of channel for an $U E_{i}$ is changed (the value of MCS for $U E_{i}$ is changed) and the $U E_{i}$ belongs to a multiplexing group at $i^{t h}$ TTI. In this case, eNodeB will send a new setup-group PDCCH that indicates the new Group_RNTI for this UE.

\section{Numerical analysis}

To estimate the performance of our method, we compare the number of required PDCCH channels of the FD scheduling and the number of required PDCCH channels of the proposed method.

According to [7] the average of data packets and PDCCH channels that $n$ active UEs require in one $m s$ can be calculated by formula 1 :

$$
A v \cdot R q L T E=\lambda\left[\frac{n v}{I_{1}}+\frac{n(1-v)}{I_{2}}\right]
$$

In which:

- $\mathrm{n}$ : number of active users

- $\lambda$ : average tranmission number

- $I_{1}$ : the inter-arrival time of voice packets

- $I_{2}$ : the inter-arrival time of SID packets

- $\mathrm{v}$ : voice activity factor

Assume that $t m s$ is the average of call time so the average required data packets and PDCCH channels of FD scheduling can be calculated by the formula as follows:

$$
A v \cdot R q F D=t \cdot A v \cdot R q L T E
$$

Suppose that the Multi-users Multiplexing Radio Voice Transmission method can classify the $A v \cdot R q F D$ require packets into $l$ multiplexing groups. UEs of $i^{t h}$ multiplexing group belong to $k_{i}$ group RNTI. So the number of required PDCCH channels in $\mathrm{t} m s$ of the proposed scheduling can be calculated by formula 3 :

$$
\text { No.GroupRNTI_CCH }=n+\sum_{k=1}^{l} k_{i} p_{i}+\epsilon
$$

Where:

- n: number of required PDCCH channels for the initilization of group

- $\epsilon$ : number of PDCCH for UEs that change group RNTI during t $m s$

- $p_{i}$ : coefficient variation size of PDCCH caused by the new adding fields

Therefore, the average of PDCCH channels that $n$ UEs require in one $m s$ can be calculated by formula 4 :

$$
\text { Av.RqGroup } R N T I=\frac{n+\sum_{k=1}^{l} k_{i} p_{i}+\epsilon}{t}
$$

The control capacity gain is given by:

$$
\text { controlgain }=\frac{A v \cdot R q L T E-A v \cdot R q G r o u p R N T I}{A v \cdot R q L T E} * 100 \%
$$

The term blocking rate refers to the number of VoIP can not be served due to the lack of data or control resources. Assume that $n_{B W}$ is number of allowed Resource Block (RB), $u_{F D R B}$ is the average of used RBs for transmitting one voice payload in the standard LTE, $u_{N e w R B}$ is the average of used RBs for transmitting one voice payload in the proposed method. The average number of data packets that can be transmitted in one TTI in the standard LTE Av.SpLTE can be calculated by formula 6 :

$$
A v \cdot S p L T E=\frac{n_{B W}}{u_{F D R B}}
$$

The average number of data packets that can be transmitted in one TTI in the proposed method Av.SpGroupRNTI can be calculated by formula 7 :

$$
\text { Av.SpGroupRNTI }=\frac{n_{B W}}{u_{N e w R B}}
$$

The data blocking rate of the standard LTE $\left(\right.$ dblock $\left._{L T E}\right)$ is calculated by the formula as follows:

$$
\text { dblock }_{L T E}=\left\{\begin{array}{l}
0 \text { if } C_{1} \\
\text { else } \frac{A v \cdot R q L T E-A v \cdot S p L T E}{A v \cdot R q L T E}
\end{array}\right.
$$

Where:

$$
\text { - } C_{1}: A v \cdot R q L T E \leq A v \cdot S p L T E
$$

Assume that in one TTI, the average number of supported PDCCH channels is Av.SpPDCCH. The control blocking rate of the standard LTE $\left(\right.$ cblock $\left._{L T E}\right)$ is calculated by the formula as follows:

$$
\text { cblock }_{L T E}=\left\{\begin{array}{l}
0 \text { if } C_{2} \\
\text { else } \frac{A v \cdot R q L T E-A v \cdot S p P D C C H}{A v \cdot R q L T E}
\end{array}\right.
$$


- $C_{2}: A v \cdot R q L T E \leq A v \cdot S p P D C C H$

The blocking rate of the standard LTE $\left(\delta_{L T E}\right)$ is calculated by the formula as follows:

$$
\delta_{L T E}=\operatorname{Max}\left(\text { dblock }_{L T E}, \text { cblock }_{L T E}\right)
$$

The data blocking rate of the proposed method $\left(d b l o c k_{G r o u p R N T I}\right)$ is calculated by the formula as follows:

$$
\text { dblock }_{\text {GroupRNTI }}=\left\{\begin{array}{l}
0 \text { if } C_{3} \\
\text { else } \frac{A v \cdot R q L T E-A v \cdot S p G r o u p R N T I}{A v \cdot R q L T E}
\end{array}\right.
$$

Where:

- $C_{3}: A v \cdot R q L T E \leq A v \cdot S p G r o u p R N T I$

The control blocking rate of the proposed method $\left(\right.$ cblock $\left._{\text {GroupRNTI }}\right)$ is calculated by the formula as follows:

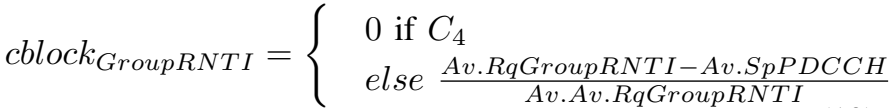

Where:

- $C_{4}: A v \cdot R q G r o u p R N T I \leq A v . S p P D C C H$

The blocking rate of the proposed method $\left(\delta_{\text {GroupRNTI }}\right)$ is calculated by the formula as follows:

$$
\delta_{\text {GroupRNTI }}=\operatorname{Max}\left(\text { dblock }_{\text {GroupRNTI }}, \operatorname{cblock}_{\text {GroupRNTI }}\right)
$$

\section{Performance EVAluation}

\section{A. Simulation Parameters}

In our context, we study and evaluate LTE as a candidate for future PMR. In PMR, the voice capacity is one of the most important factor, therefore the voice codec with low bit rate is commonly used. In our simulation, the AMBE codec with low bit rate $(2450 \mathrm{bps})$, the low bandwidths of LTE (1.4 MHz, 3 $\mathrm{MHz}, 5 \mathrm{MHz}$ ), 24 bits CRC and Normal Cyclic Prefix are used for our evaluation. We compare the blocking rate of the Fully Dynamic Scheduling and the proposed method for different number of active users $(50,100,200,500,1000,2000)$ having random MCS values. The main simulation parameters are presented in Table III.

\section{B. Simulation Results}

Figure 3 shows the comparison of required number of PD$\mathrm{CCH}$ channels in one TTI between Fully Dynamic scheduling and the proposed method for different number active users in case of $1.4 \mathrm{MHz}$ bandwidth. It is apparent that there was a significant reduction of the required number of PDCCH channels of the proposed method in comparison to the Fully Dynamic scheduling especially in case of the high number of active users. In the best case, the proposed method can reduce the control overhead up to $85 \%$. On average, the reduction of control overhead is about $65 \%$.

Figures 4, 5 and 6 compare the blocking rate of the Fully Dynamic scheduling and the proposed method for the coresponding $1.4 \mathrm{MHz}$ bandwidth, $3 \mathrm{MHz}$ bandwidth and $5 \mathrm{MHz}$
TABLE III

SIMULATION PARAMETERS

\begin{tabular}{|l|l|}
\hline Parameter & Value \\
\hline Voice codec & AMBE 2450 bps \\
\hline Frame structure & Type 1 FDD \\
\hline Bandwidth & $1.4 \mathrm{MHz}, 3 \mathrm{MHz}, 5 \mathrm{MHz}$ \\
\hline Symbols for PDCCH & 3 symbols \\
\hline CRC & 24 bits \\
\hline Number of active UEs & $50,100,200,500,1000,2000$ \\
\hline Cyclic Prefix & Normal \\
\hline Number of antenna & 2 \\
\hline Average time call & $60 \mathrm{~s}$ \\
\hline$\lambda$ & 1.2 \\
\hline$I_{1}$ & 20 \\
\hline$I_{1}$ & 160 \\
\hline $\mathrm{v}$ & $50 \%$ \\
\hline$p_{i}$ & 2 \\
\hline CCEs for Downlink & $50 \%$ of total CCEs \\
\hline
\end{tabular}

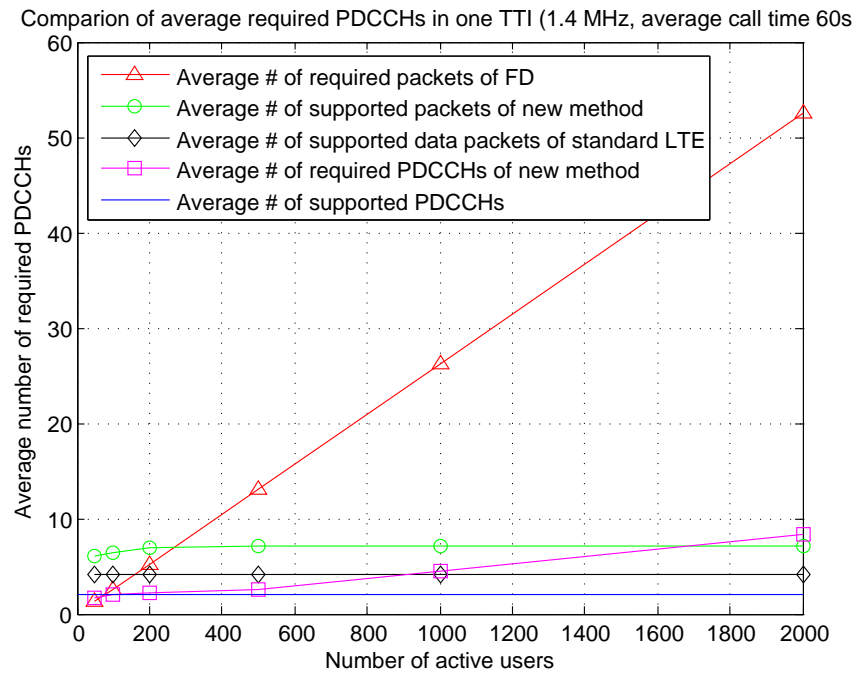

Fig. 3. Comparison of number of required PDCCH channels between Fully Dynamic scheduling and the proposed method in one TTI for $1.4 \mathrm{MHz}$ bandwidth

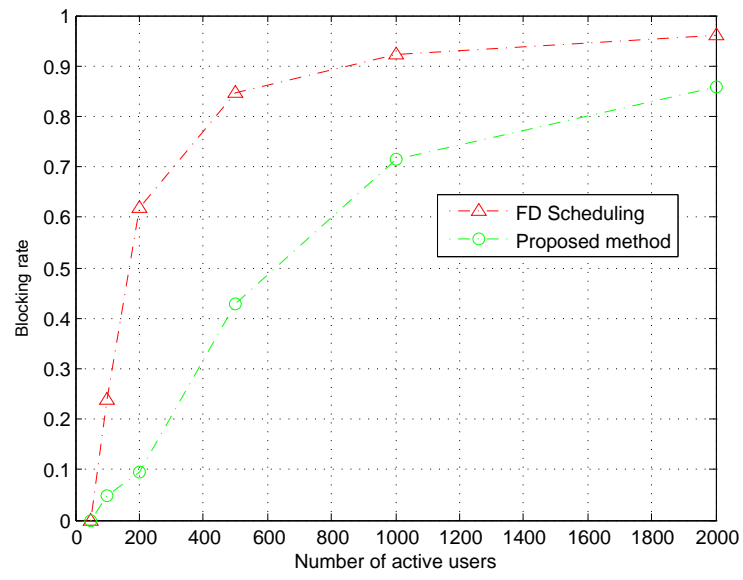

Fig. 4. Comparison of blocking rate between Fully Dynamic scheduling and the proposed method for $1.4 \mathrm{MHz}$ bandwidth 


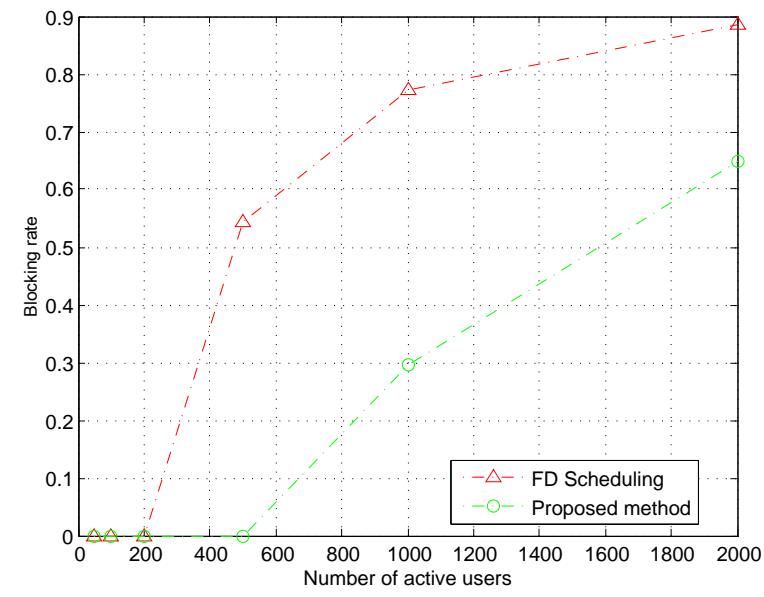

Fig. 5. Comparison of blocking rate between Fully Dynamic scheduling and the proposed method for $3 \mathrm{MHz}$ bandwidth

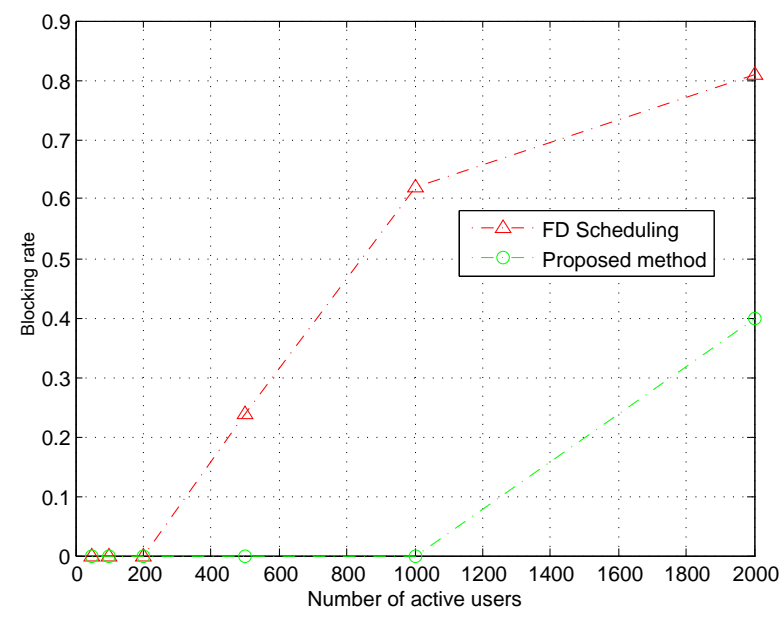

Fig. 6. Comparison of blocking rate between Fully Dynamic scheduling and the proposed method for $5 \mathrm{MHz}$ bandwidth

bandwidth. The results show that the higher the bandwidth is, the higher efficiency the proposed method gains. In addition, with each bandwidth, the higher number of active UEs in the cell, the higher voice capacity gain our method can obtain.

\section{CONCLUSION}

In this paper, we presented a new Group Radio Network Temporary Identifier (Group_RNTI) method for reducing the control overhead in the Multi-users Multiplexing Radio Voice
Transmission scheme. Our method clusters UEs having the same MCS into one or more groups. Each group is assigned a common Group_RNTI value. The Group_RNTI values are used for the scheduling to reduce the number of required PD$\mathrm{CCH}$ channels in the multiplexing scheme. The results show that our proposed method can reduce the control overhead up to $85 \%$. In addition, the greater number of UEs in the cell and the higher bandwidth, the higher the voice capacity gain and the lower blocking rate our method can obtain. The use of Group_RNTI for the Multi-users Multiplexing Radio Voice Transmission method allows reducing both data overhead and control overhead issues for VoLTE in PMR context. Therefore, the proposed method increases the voice capacity and reduces the blocking rate of VoLTE in PMR context.

\section{REFERENCES}

[1] MC Nguyen, H Nguyen, E Georgeaux, P Mege, L Martinot, and A Florea. Architecture for Multi-users Multiplexing Radio Voice Transmission for Enhancing Voice Capacity over LTE in PMR Context. Personal, Indoor and Mobile Radio Communications (PIMRC), 2014.

[2] MB Simic. Feasibility of long term evolution (lte) as technology for public safety. In Telecommunications Forum (TELFOR), 2012 20th, pages 158-161. IEEE, 2012.

[3] Xavier Pons Masbernat, Christophe Gruet, Frédéric Fraysse, Serge Contal, and Lirida Naviner. Green solutions for future lte pmr networks. In Computer Aided Modeling and Design of Communication Links and Networks (CAMAD), 2012 IEEE 17th International Workshop on, pages 165-167. IEEE, 2012.

[4] C Bormann, C Burmeister, M Degermark, H Fukushima, H Hannu, LE Jonsson, R Hakenberg, T Koren, K Le, Z Liu, et al. RObust Header Compression (ROHC): Framework and four profiles: RTP, UDP, ESP, and uncompressed. Technical report, RFC 3095, July, 2001.

[5] ER Vale, MA Grivet, and JC Brandao. Reducing the VoIP packet overhead in UMTS air interface. In Adaptive Science \& Technology (ICAST), 2009.

[6] AA Florea, H Nguyen, L Martinod, and C Molko. Serial Progressive Hierarchical Turbo Codes. In Global Telecommunications Conference (GLOBECOM), 2011.

[7] D Jiang, H Wang, E Malkamaki, and E Tuomaala. Principle and performance of semi-persistent scheduling for VoIP in LTE system. In Wireless Communications, Networking and Mobile Computing (WiCom), 2007.

[8] Sean McBeath, Jack Smith, Doug Reed, Hao Bi, Danny Pinckley, Alfonso Rodriguez-Herrera, and Jim O'Connor. Efficient signaling for VoIP in OFDMA. In Wireless Communications and Networking Conference (WCNC), 2007.

[9] Shweta Shrivastava and Rath Vannithamby. Group scheduling for improving voip capacity in ieee $802.16 \mathrm{e}$ networks. In Vehicular Technology Conference, 2009. VTC Spring 2009. IEEE 69th, pages 1-5. IEEE, 2009.

[10] Haiming Wang and Dajie Jiang. Performance comparison of control-less scheduling policies for voip in lte ul. In Wireless Communications and Networking Conference, 2008. WCNC 2008. IEEE, pages 2497-2501. 